\title{
Classical origin of conductance oscillations in an integrable cavity
}

DOI:

10.1103/PhysRevB.94.195304

Document Version

Final published version

Link to publication record in Manchester Research Explorer

\section{Citation for published version (APA):}

Pöltl, C., Kozikov, A., Ensslin, K., Ihn, T., Jalabert, R. A., Reichl, C., Wegscheider, W., \& Weinmann, D. (2016). Classical origin of conductance oscillations in an integrable cavity. Physical Review B, 94(19), [195304]. https://doi.org/10.1103/PhysRevB.94.195304

\section{Published in:}

Physical Review B

\section{Citing this paper}

Please note that where the full-text provided on Manchester Research Explorer is the Author Accepted Manuscript or Proof version this may differ from the final Published version. If citing, it is advised that you check and use the publisher's definitive version.

\section{General rights}

Copyright and moral rights for the publications made accessible in the Research Explorer are retained by the authors and/or other copyright owners and it is a condition of accessing publications that users recognise and abide by the legal requirements associated with these rights.

\section{Takedown policy}

If you believe that this document breaches copyright please refer to the University of Manchester's Takedown Procedures [http://man.ac.uk/04Y6Bo] or contact uml.scholarlycommunications@manchester.ac.uk providing relevant details, so we can investigate your claim.

\section{OPEN ACCESS}




\title{
Classical origin of conductance oscillations in an integrable cavity
}

\author{
Christina Pöltl, ${ }^{1}$ Aleksey Kozikov, ${ }^{2,}{ }^{*}$ Klaus Ensslin, ${ }^{2}$ Thomas Ihn, ${ }^{2}$ Rodolfo A. Jalabert, ${ }^{1}$ Christian Reichl, ${ }^{2}$ \\ Werner Wegscheider, ${ }^{2}$ and Dietmar Weinmann ${ }^{1}$ \\ ${ }^{1}$ Institut de Physique et Chimie des Matériaux de Strasbourg, Université de Strasbourg, CNRS UMR 7504, 23 rue du Loess, \\ Boîte Postale 43, F-67034 Strasbourg, France \\ ${ }^{2}$ Solid State Physics Laboratory, ETH Zürich, CH-8093 Zürich, Switzerland \\ (Received 21 July 2016; revised manuscript received 4 October 2016; published 7 November 2016)
}

\begin{abstract}
Scanning gate microscopy measurements in a circular ballistic cavity with a tip placed near its center yield a nonmonotonic dependence of the conductance on the tip voltage. Detailed numerical quantum calculations reproduce these conductance oscillations, and a classical scheme leads to its physical understanding. The largeamplitude conductance oscillations are shown to be of classical origin, and they are well described by the effect of a particular class of short trajectories.
\end{abstract}

DOI: 10.1103/PhysRevB.94.195304

\section{INTRODUCTION}

The scanning gate microscopy (SGM) technique [1-8] has been developed and applied to study two-dimensional electron gases (2DEGs) surrounding a quantum point contact (QPC) and other mesoscopic systems [7,9]. Initially, the goal was to obtain information beyond that provided through standard quantum transport experiments by measuring the effect of a local potential on the sample conductance. However, the interpretation of the SGM measurements remains challenging $[7,8,10,11]$, in particular because most experiments operate in a regime where the potential induced by the SGM tip strongly perturbs the 2DEG. Performing SGM with tunable geometries indicated that the presence of confinement has to be taken into account in the interpretation of the data $[12,13]$.

A more recent purpose of SGM is the usage of the tip to control and modify the potential landscape, and thus the sample geometry, allowing systematic studies of the effect of sample shape on coherent electron transport. For the example of a circular cavity connected to leads by QPCs [14], the tip has been used to control and study magnetoelectric subbands. Another example along these lines is the electronic analog of the Braess paradox $[15,16]$, where the tip is used to cut one out of several routes of electron transport through the sample.

While the signatures of an underlying classically chaotic electron dynamics have been clearly established [17], the situation with integrable geometries is less conclusive due to the lack of global stability of the dynamics and the unavoidable effect of smooth disorder in the samples. In particular, the conductance fluctuations and weak localization in circular cavities have been studied experimentally and compared with other geometries [18-22]. Intriguingly, the observed behavior did not always correspond to what was expected for an integrable geometry. Controlling the potential landscape of a ballistic cavity with an SGM tip allows one to alter the underlying classical dynamics within a given sample, and

\footnotetext{
*Present address: School of Physics and Astronomy, University of Manchester, Oxford Road, Manchester M13 9PL, UK.
}

thus to compare different classical dynamics within the same sample.

In this paper, we present SGM measurements on a circular cavity exhibiting an unexpected nonmonotonic dependence of the conductance through the cavity on the strength of a tip placed over the center. Detailed numerical modeling of the measured structure reproduced these large-amplitude conductance oscillations with tip strength. To understand the physical mechanism at the origin of the conductance oscillations with tip strength, we develop a semiclassical approach that demonstrates the key role played by the modification of classical trajectories induced by the tip potential. The statistical analysis of the ensemble of classical trajectories leads to a detailed understanding of the underlying mechanism and the crucial role played by the smoothness of the tip potential.

In Sec. II we present the SGM measurements on a circular cavity, and in particular the nonmonotonic dependence of the conductance on tip strength when the tip is placed near the center. The numerical simulation of the conductance as a function of the strength of a tip placed in the cavity center is shown in Sec. III for a realistic model that succeeds in providing a quantitative description of the measured conductance. In Sec. IV we present a simplified model possessing nevertheless the essential ingredients to yield the conductance oscillations, in qualitative agreement with those of the experiment. Section $\mathrm{V}$ discusses the evaluation of the conductance based on classical trajectories. The statistical analysis of the ensemble of trajectories in Sec. VI provides a classical understanding of the conductance oscillations, and a simplified treatment presented in Sec. VII highlights the basic mechanism behind the effect. In Sec. VIII we compare experimental results beyond the case of a centered tip with the numerics and a classical estimate for the size of the region where the conductance varies nonmonotonically with tip strength.

\section{EXPERIMENTAL OBSERVATION}

The SGM response has been measured in a circular ballistic cavity with a diameter of about $3 \mu \mathrm{m}$, electrostatically defined in a GaAs-GaAlAs heterostructure. The chosen setup is such that the cavity is connected to source and drain by wide 
(a)
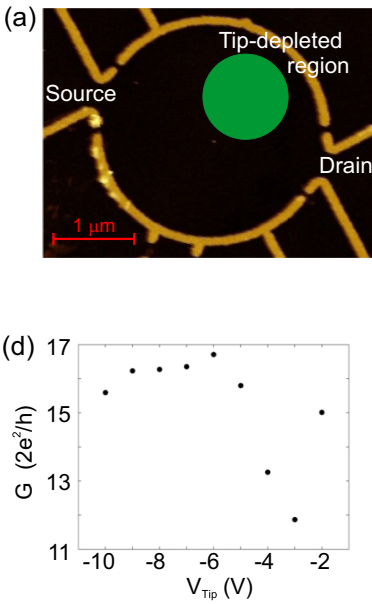

(b)

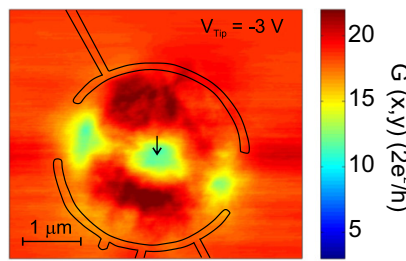

(c)

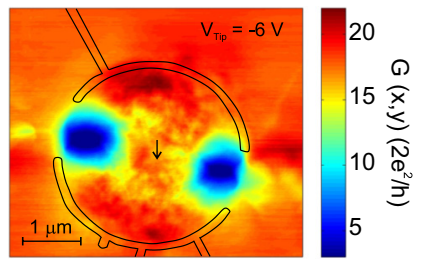

FIG. 1. (a) AFM image of the cavity used in the experiment. The black area corresponds to the surface of GaAs. Yellow contacts are top gates. The tip-induced depleted region is indicated by the green circle for $V_{\text {tip }} \approx-8 \mathrm{~V}$. The current flows between source and drain contacts. (b) and (c) Conductance $G$ as a function of tip position at a tip voltage of -3 and $-6 \mathrm{~V}$, respectively. Black lines outline biased top gates (the grounded gates are not shown). (d) Tip voltage dependence of the conductance when the tip is at the center of the cavity [marked by an arrow in (b) and (c)].

openings having a width of about $1 \mu \mathrm{m}$. The 2DEG is $120 \mathrm{~nm}$ below the surface, with a transport mean free path of $49 \mu \mathrm{m}$. The Fermi energy is $E_{\mathrm{F}}=4.3 \mathrm{meV}$ and the Fermi wavelength is about $\lambda_{\mathrm{F}}=72 \mathrm{~nm}$. The size of the structure, being much smaller than the elastic mean free path, and the low temperatures used in the experiment set the present study in the coherent ballistic regime.

Figure 1(a) shows an atomic force microscopy (AFM) image of the sample where top gates (yellow) are placed on the surface of the structure. The effect of the SGM tip is controlled by the tip voltage $V_{\text {tip }}$. For $V_{\text {tip }} \lesssim-3.5 \mathrm{~V}$, the tip potential creates a depletion disk in the 2 DEG whose size increases with increasingly negative tip voltages. The green circle indicates the approximate size of the depletion disk at $V_{\text {tip }} \approx-8 \mathrm{~V}$. Figures 1 (b) and 1(c) show the conductance through the cavity as a function of tip position for fixed values of $V_{\text {tip. }}$. The circular cavity is defined by sufficiently negative voltages applied to the top gates indicated by the black lines. The other gates visible in (a) are grounded, resulting in a very open cavity. When the tip is placed close to the entrances of the cavity, there is a suppression of the conductance that becomes more pronounced for more negative tip voltages.

A special situation arises when the tip is close to the center. For the less negative tip voltage (b), the conductance is suppressed by the effect of the tip, while for the more negative $V_{\text {tip }}$ (c), we observe an enhancement of the conductance. Figure 1(d) shows the conductance as a function of $V_{\text {tip }}$ with the position of the tip fixed at the center of the cavity. The nonmonotonic dependence of the conductance on the tip strength leads to a surprising observation: Going for a more invasive configuration with larger depletion disks and further blocking of the area for electron transport may result in an enhancement of the conductance [23].

To achieve a theoretical understanding of the intriguing behavior of the conductance observed in the experiment (Fig. 1), we perform numerical simulations of models of different complexity and develop a semiclassical approach, focusing on the situation in which the tip is in the center of the cavity.

\section{NUMERICAL SIMULATION OF THE EXPERIMENT}

Attempting a quantitative description of electronic transport through a microstructure is a challenging task, due to the unknown features of the self-consistent electronic potential at the basis of one-particle modeling. We start by considering a disorder-free circular cavity with a tip in its center, using parameters and conditions that are as close as possible to those of the real sample. The electrostatic confinement potential of the cavity due to the charged top gates is calculated using COMSOL with the exact geometry parameters of the sample shown in Fig. 1. We take the standard approach [24] of modeling the SGM tip by a Lorentzian potential,

$$
U_{\mathrm{S}}(\vec{r})=\frac{u_{\mathrm{t}} A}{w_{\mathrm{t}}^{2}+\left[\vec{r}-\vec{r}_{\mathrm{T}}\right]^{2}} .
$$

The constant $A=E_{\mathrm{F}} R_{0}^{2}$ is introduced in order to work with a dimensionless tip-strength parameter $u_{\mathrm{t}}$. We choose $E_{\mathrm{F}}$ as the energy scale and the radius of the cavity $R_{0}=1500 \mathrm{~nm}$ as the length scale [25]. The width $w_{\mathrm{t}}=200 \mathrm{~nm}$ leads to a realistic tip size, of the order of the tip-2DEG distance. In most of our analysis, the tip position $\vec{r}_{\mathrm{T}}$ is fixed at the center of the cavity.

The coherent zero-temperature conductance was computed with KWANT [26], a package that implements a recursive Green function algorithm. A tight-binding square lattice is used with a lattice parameter $a=5 \mathrm{~nm}$, much smaller than $\lambda_{\mathrm{F}}$. The dimensionless conductance $g=G /\left(2 e^{2} / h\right)$ is presented and discussed henceforth. The corresponding numerical results are shown in Fig. 2(a). Superposed to the small-scale ballistic conductance fluctuations, one observes a large-scale oscillation of the conductance with tip strengths for weak and moderately strong tips. Such a behavior reproduces the experimentally measured nonmonotonic SGM response.

To relate the tip strength parameter $u_{\mathrm{t}}$ appearing in the model potential (1) with the tip voltage $V_{\text {tip }}$ used to control the tip strength in the experiment, we follow the procedure described in Ref. [27] to estimate the size of the depletion disk from the SGM response when the tip is close to the cavity edges or a QPC. Choosing the value of $u_{\mathrm{t}}$ such that the depletion disk of the Lorentzian potential (1) corresponds to the estimated size, we find the approximately linear relation between the tip strength $u_{\mathrm{t}}$ and the voltage $V_{\text {tip }}$ shown in the inset of Fig. 2(a). We extrapolate the relation down to weak tip potentials where no depletion occurs. Using such a rough estimate, we are able to convert the experimental data of Fig. 1(d) in order to present them in Fig. 2(a) as a function of tip strength $u_{\mathrm{t}}$, together with the numerically obtained conductance. Besides small deviations in tip strength that may be due to uncertainties in the precise shape of the tip potential, the agreement is good, confirming the quantitative validity of our model for the description of the experimental setup.

It can be noticed that the second conductance maximum, obtained in the simulations around $u_{\mathrm{t}}=0.27$, is beyond the 


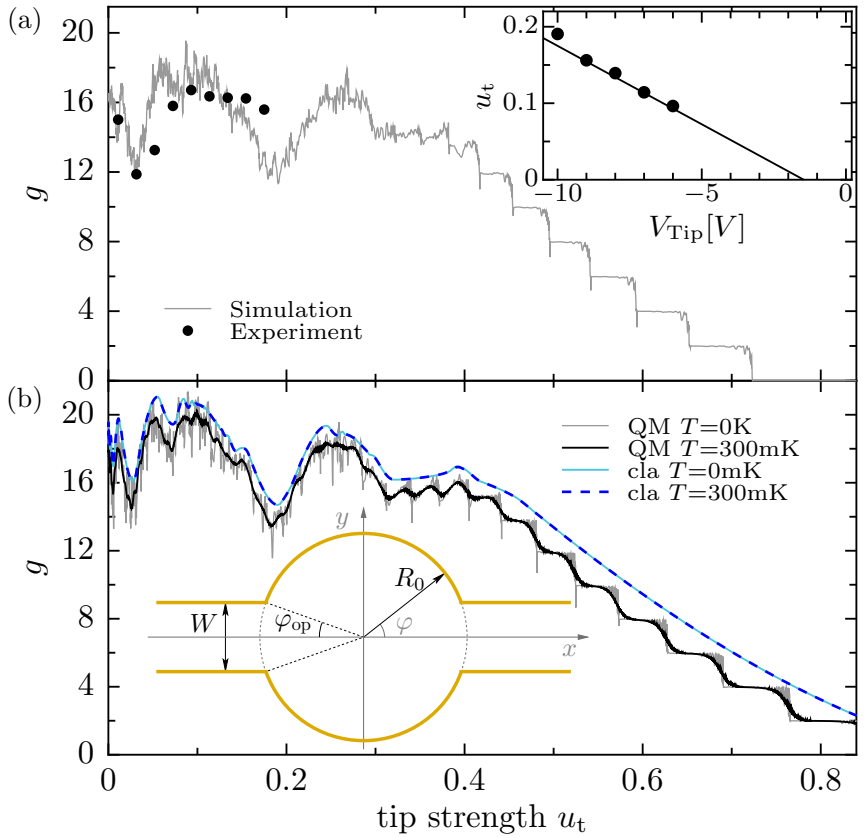

FIG. 2. Dimensionless conductance as a function of the tip strength $u_{\mathrm{t}}$ for (a) the realistic model described in Sec. III and (b) the simplified model treated in Sec. IV. Gray solid lines are for the coherent zero-temperature conductance. In (a), the dots correspond to the experimental points of Fig. 1(d) using the linear identification between $V_{\text {tip }}$ and $u_{\mathrm{t}}$ shown in the inset of (a) and discussed in the text. In (b), the black line represents the quantum conductance at $T=300 \mathrm{mK}$. The light blue and dark blue (dashed) lines are the classical results at $T=0$ and $300 \mathrm{mK}$, respectively. Inset: sketch of the considered setup. A circular cavity with radius $R_{0}$ is connected to quasi-one-dimensional leads of width $W, \varphi$ measures the angles from the origin, and $2 \varphi_{\mathrm{op}}$ is the opening angle of the contacts seen from the center of the cavity.

available experimental data. In the regime of very strong tip potentials, the conductance exhibits plateaus at conductance values that are multiples of $2 \times\left(2 e^{2} / h\right)$, which decrease with increasing tip strength. Such a behavior occurs in the regime where the depletion disk generated by the tip becomes so large that the device is reminiscent of two parallel quantum wires having the same quantized conductance.

The current density is a local quantity of major interest in SGM studies [3,11]. In the invasive regime of the experiments, the current density in the cavity depends strongly on the tip strength. In Fig. 3, the current density calculated from scattering states at the Fermi energy that are impinging from the left lead is shown as a function of the position within the cavity, for a tip placed in the center with strength $u_{\mathrm{t}}=0.128$ [close to the first maximum of the large-scale oscillations seen in Fig. 2(a)]. The central (black) area of vanishing current density reflects the tip-depleted area. The diamondlike pattern observed in the current flow around the tip suggests the signature of classical electron trajectories following these lines.

The agreement obtained between the experimental measurements and the numerical results in the realistic system (together with the current density plots such as that of Fig. 3) constitutes a detailed level of description of quantum transport,

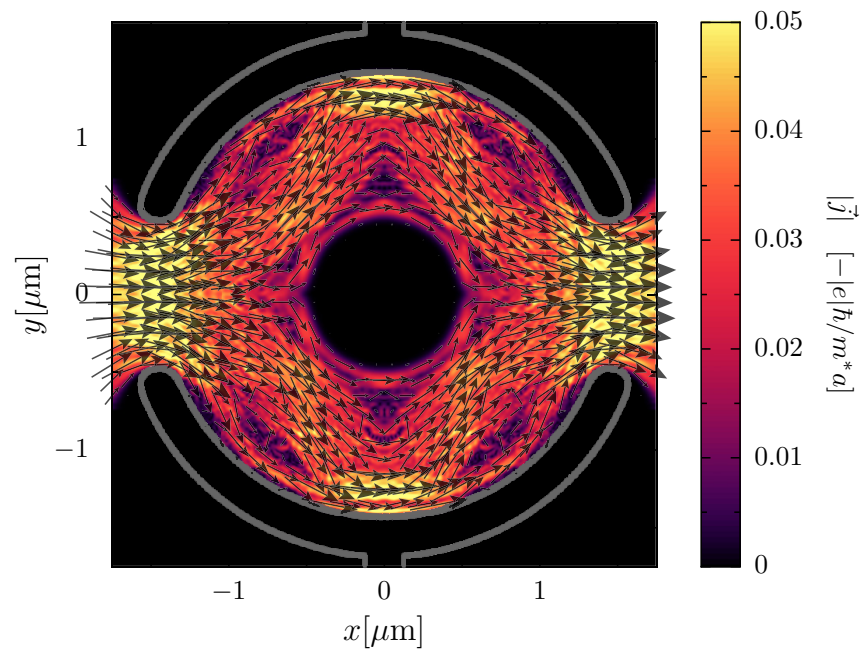

FIG. 3. Current density of scattering states impinging from the left through the realistic cavity of Fig. 2(a), with a tip of strength $u_{\mathrm{t}}=0.128$ at the center. The color scale gives the absolute value of the local current, and thin black arrows reflect the current direction. The thick gray lines indicate the region where the confinement potential depletes the 2DEG.

but it does not by itself enable us to reach an understanding of the physical mechanism behind the observed conductance oscillations. The next step toward such a goal, made in the following section, consists in elaborating a simplified model that exhibits conductance oscillations and, at the same time, allows for the identification and analysis of the classical trajectories.

\section{CONDUCTANCE OSCILLATION IN A SIMPLIFIED MODEL SYSTEM}

To understand the mechanisms underlying the nonmonotonic tip dependence of the conductance and to find the key ingredients for the occurrence of the phenomenon, we should attempt to reduce the complexity of the model. Though it is tempting to assume hard-wall boundaries for the cavity and for the tip potential, no significant large-scale conductance oscillations occur within such a model. Our analysis in Sec. VII provides an explanation for the failure of a fully hard-wall model to yield conductance oscillations.

We found that a hard-wall cavity attached to semi-infinite leads as sketched in the inset of Fig. 2(b), perturbed by a tip potential of the form

$$
U_{\mathrm{M}}(\vec{r})=\frac{u_{\mathrm{t}} A}{\left(\vec{r}-\vec{r}_{\mathrm{T}}\right)^{2}},
$$

is a simplified, minimal model that reproduces all the features observed in the realistic simulation [28]. An important advantage of this model is that the classical trajectories can be found analytically. The parameter $A$ is defined after Eq. (1). In this model, we took $R_{0}=1500 \mathrm{~nm}$ as the radius of the cavity and $W=1000 \mathrm{~nm}$ for the width of the leads.

Figure 2(b) shows the conductance as a function of tip strength $u_{\mathrm{t}}$ for the simplified model. The gray solid line shows the coherent quantum conductance at zero temperature. The black line is the coherent conductance at $T=300 \mathrm{mK}$, 
obtained from a convolution of the energy-dependent zerotemperature conductance with the derivative of the Fermi distribution, while the light blue and dark blue (dashed) lines represent the results obtained from our analysis based on classical trajectories (see Sec. V for details). The main features of the zero-temperature quantum conductance are the same in the realistic simulation and in the simplified model.

For large tip strength, the conductance decreases in quantized steps of height $2 \times\left(2 e^{2} / h\right)$ as in the realistic model. For weaker tip strength, large-scale oscillations with an amplitude of about $6 \times\left(2 e^{2} / h\right)$ dominate the tip dependence of the conductance. Strikingly, and even though the tip potential represents a repulsive obstacle for the electrons, at some tip strengths the conductance even exceeds that of the unperturbed cavity. Superposed with this secular behavior, the zero-temperature conductance exhibits ballistic conductance fluctuations with an amplitude of about $2 \times\left(2 e^{2} / h\right)$. Those conductance fluctuations are expected to decay with temperature on a scale that corresponds to the correlation energy of the fluctuations. In classically chaotic systems, the latter can be estimated within a semiclassical approach $[29,30]$ to be of the order of $\hbar / \tau=\hbar v_{\mathrm{F}} /\langle L\rangle$, where $\tau$ is the average time an electron spends in the cavity, $\langle L\rangle$ is the average length of classical trajectories through the cavity, and $v_{\mathrm{F}}$ is the Fermi velocity. Assuming $\langle L\rangle \approx W / \pi R_{0}$ and using the chaotic prediction with the parameters of our system yields a temperature scale of about $75 \mathrm{mK}$. Consistent with such an estimate, we find numerically that the fluctuations are indeed suppressed at the temperature of $T=300 \mathrm{mK}$ that is used in the experiment, thereby confirming their quantum origin. In contrast, the larger-scale oscillations remain robust, pointing to a different mechanism determining their occurrence. We show in the following section that they are of classical origin.

\section{ANALYSIS IN TERMS OF CLASSICAL TRAJECTORIES}

In the ballistic regime, the conductance in the classical limit of $\hbar \rightarrow 0$ (where quantum interference is suppressed) can be expressed in terms of classical trajectories traveling between the entrance and exit of the structure as $[17,31]$

$$
g_{\text {class }}=\frac{m v_{0} W}{\hbar \pi} \mathcal{T}
$$

up to a constant whose value is not accessible by the semiclassical approach leading to Eq. (3) and which we will ignore. The quantities $m$ and $v_{0}$ are, respectively, the mass and initial velocity of the electrons at the Fermi energy. The factor $m v_{0} W /(\hbar \pi)$ stands for the incoming electron flux, and its integer part is the number of propagating channels in the leads. For the case of GaAs, we use $m=0.067 m_{0}$, with $m_{0}$ the free-electron mass. The transmission probability is given by

$$
\mathcal{T}=\frac{1}{2} \int_{-\pi / 2}^{\pi / 2} d \theta \cos \theta \int_{-W / 2}^{W / 2} d y f(y, \theta),
$$

where $f(y, \theta)=1(0)$ for transmitted (reflected) trajectories that enter the cavity at a cross section in the left contact at $y$ and with the momentum direction characterized by the angle $\theta$ with respect to the $x$ axis [32]. In quantum billiards, where the electrostatic potential is either zero or infinity, the trajectories depend on the geometry but not on the electron energy. Therefore, $\mathcal{T}$ is independent of the energy and can be obtained from the asymptotic values of the quantum conductance in the limit of infinite energy. In the case under study, we do not have a quantum billiard, due to the smooth character of the tip potential, and therefore $\mathcal{T}$ is energy-dependent.

Equation (3) for a ballistic system is the equivalent of the Drude conductance for the disordered case. In ballistic structures, the smooth disorder only weakly affects trajectories that are considerably shorter than the transport mean free path. Therefore, if the properties under study are dominated by the contribution of short trajectories, it is possible to ignore the disorder altogether and to include only the trajectories of the clean (disorder-free) geometry.

The function $f(y, \theta)$ is generally difficult to determine, and the integrals in Eq. (4) are typically calculated by randomly sampling the initial conditions. However, the case of a hard-wall circular cavity with a central tip represented by the potential (2) is quite simple due to the conservation of angular momentum within the cavity and the availability of an analytical expression describing the trajectories.

A trajectory is determined by the initial conditions of a particle in the left contact, described by its energy, position, and momentum orientation. The analytical expression of trajectories in the potential (2) and specular reflection laws allow us to determine the subsequent points where the electron hits the circle of radius $R_{0}$ representing the cavity wall [see the inset of Figs. 2(b) and Fig. 4]. As soon as such a point lies in one of the contacts between the cavity and the leads (i.e., $|y|<W / 2$ ), the trajectory is completed and contributes to the transmission (reflection) probability if it reaches the right (left) exit. Figure 4 shows a few examples of transmitted (blue solid) and reflected (red dashed) trajectories at different tip strength, which are discussed below.

The description of a trajectory can be reduced to an equidistant series of position angles $\varphi_{n}=\varphi_{0} \pm n \Delta \varphi$ where the particle hits the cavity edge for trajectories turning counterclockwise (clockwise) around the cavity center. We denote by $\varphi_{0}$ the initial angle with respect to the $x$ axis describing the starting point in the left contact [see Fig. 4(a) for a sketch], which is taken on the dashed line at radius $r=R_{0}$ [33], and $\Delta \varphi$ is the (positive) rotation angle, which is a characteristic parameter of the trajectory.

The classical results shown in Fig. 2(b) are based on the application of Eq. (3) in the framework of the simplified model. For each value of $u_{\mathrm{t}}$, we sampled a finite ensemble of trajectories $\mathcal{M}$ corresponding to different initial conditions (at fixed energy) in the left contact. The prescription [31] to calculate the classical transmission using Eqs. (3) and (4) is implemented adopting homogeneously distributed transverse starting points and, since $d \theta \cos \theta=d(\sin \theta)$, a sampling of initial angles $\theta$ such that the values of $\sin \theta$ are equally spaced. Such a sampling yields the expression

$$
g_{\text {class }}=\frac{m v_{0} W}{\hbar \pi} \frac{\# \mathcal{M}_{\mathrm{T}}}{\# \mathcal{M}}
$$

for the classical conductance in terms of the cardinalities (denoted by \#) of the subset of transmitted trajectories $\mathcal{M}_{\mathrm{T}}$ and that of the total set of sampled trajectories $\mathcal{M}=\mathcal{M}_{\mathrm{T}} \cup \mathcal{M}_{\mathrm{R}}$ $\left(\mathcal{M}_{\mathrm{R}}\right.$ denotes the subset of reflected trajectories). 


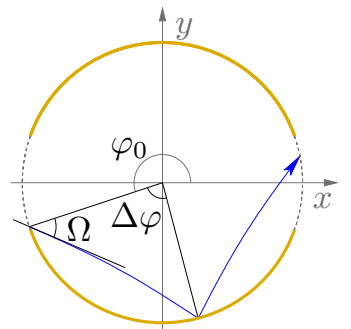

(a)

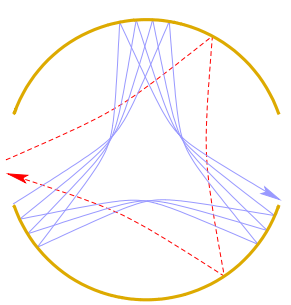

(b)

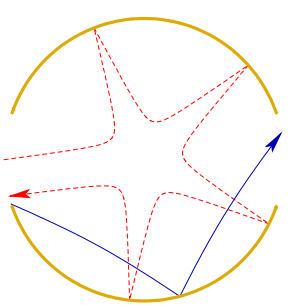

(c)

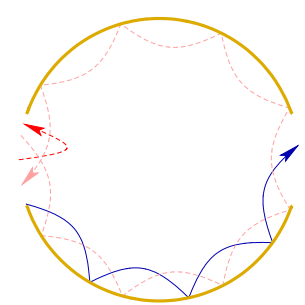

(d)

FIG. 4. (a) Definition of the coordinate system and the angles used to characterize individual trajectories. $\varphi_{0}$ is the angle of the initial position with respect to the $x$ axis, $\Omega$ denotes the angle between the initial momentum of the trajectory and the diameter of the circle, and $\Delta \varphi$ is the angular distance between two subsequent collisions with the cavity wall. Two examples of transmitted (blue solid) and reflected (red dashed) classical trajectories are shown in (b) for tip strength $u_{\mathrm{t}}=0.03$ and in (c) for $u_{\mathrm{t}}=0.06$; three trajectories are shown in (d) for a rather strong tip characterized by $u_{\mathrm{t}}=0.43$.

The classical conductance [light and dark blue dashed lines in Fig. 2(b)] has only a very weak temperature dependence (at least up to $T=300 \mathrm{mK}$ ). Its behavior is remarkably close to the finite-temperature quantum conductance in the regime of not too strong tip strength. The small offset of our classical conductance with respect to the quantum can be attributed to the ignored constant in Eq. (3). In particular, the classical results clearly exhibit the tip-strength-dependent oscillations. Their ability to describe the behavior of the numerically calculated quantum conductance demonstrates that the large-scale oscillations are of classical origin. In contrast, the conductance fluctuations and the conductance quantization for strong tip strength are quantum effects and therefore not present in the classical results. We conclude that the experimentally observed behavior at $T=300 \mathrm{mK}$ is very well described by the classical treatment of our simplified model, except for the conductance quantization at large tip strength.

In the sequel of the paper, we present a detailed analysis in order to understand why the rich variety of classical trajectories (some of them shown in Fig. 4) results in the simple structure of $g_{\text {class }}$ presented in Fig. 2. The properties of the classical trajectories through the sample and their dependence on tip strength will be analyzed in an effort to understand the mechanism that leads to the large conductance oscillations.

\section{MECHANISM LEADING TO CONDUCTANCE OSCILLATIONS}

To understand the origin of the large-scale conductance oscillations at low tip strength, we investigate the dependence of the contributing trajectories on the strength of the tip potential. We characterize each trajectory $s$ by two parameters: the number of bounces at the cavity edge $b_{s}$, and the number of windings around the cavity center $w_{s}$. We distinguish transmitted $(\alpha=\mathrm{T})$ and reflected $(\alpha=\mathrm{R})$ trajectories.

The probability that a trajectory is transmitted or reflected after $b$ bounces with the cavity edge can be calculated as

$$
P_{\alpha}^{\mathrm{b}}(b)=\frac{\sum_{s \in \mathcal{M}_{\alpha}} \delta_{b, b_{s}}}{\# \mathcal{M}}
$$

with $\alpha=\mathrm{T}$ and $\mathrm{R}$, respectively. The calculation of the probabilities of having $w$ windings $P_{\alpha}^{\mathrm{w}}(w)$ is analogous to Eq. (6). We also introduce the probabilities $P^{\mathrm{b}}(b)=\sum_{\alpha} P_{\alpha}^{\mathrm{b}}(b)$ and $P^{\mathrm{w}}(w)=\sum_{\alpha} P_{\alpha}^{\mathrm{w}}(w)$ of having a trajectory with $b$ bounces and $w$ windings, respectively.

Due to the wide openings of the cavity, most of the features of the problem at hand can be explained by only considering relatively short trajectories having few bounces with the cavity wall $\left(b_{s} \leqslant 8\right)$ and very few windings around the cavity center $\left(w_{s} \leqslant 1\right)$. This assumption is confirmed by the comparison shown in Fig. 5(a) between the full classical conductance given by Eq. (5) (black solid line) and restricted sums over trajectories with bounded numbers of bounces (dotted line) or windings (dashed lines). In particular, restricting the reflected trajectories leads to upper bounds of the conductance,

$$
\begin{aligned}
g_{b_{\max }}^{\mathrm{b},>} & =\frac{m v_{0} W}{\hbar \pi}\left(1-\sum_{b=0}^{b_{\max }} P_{\mathrm{R}}^{\mathrm{b}}(b)\right), \\
g_{w_{\text {max }}}^{\mathrm{w},>} & =\frac{m v_{0} W}{\hbar \pi}\left(1-\sum_{w=0}^{w_{\max }} P_{\mathrm{R}}^{\mathrm{w}}(w)\right),
\end{aligned}
$$

while restricting the transmitted trajectories provides lower bounds,

$$
g_{b_{\max }}^{\mathrm{b},<}=\sum_{b=0}^{b_{\max }} g^{\mathrm{b}}(b) \quad \text { and } \quad g_{w_{\max }}^{\mathrm{w},<}=\sum_{w=0}^{w_{\max }} g^{\mathrm{w}}(w),
$$

where

$$
g^{\mathrm{b}}(b)=\frac{m v_{0} W}{\hbar \pi} P_{\mathrm{T}}^{\mathrm{b}}(b) \quad \text { and } \quad g^{\mathrm{w}}(w)=\frac{m v_{0} W}{\hbar \pi} P_{\mathrm{T}}^{\mathrm{w}}(w)
$$

are the conductance contributions of trajectories with $b$ bounces and $w$ windings, respectively. The bounds $g_{8}^{\mathrm{b},>}, g_{1}^{\mathrm{w},>}$, and $g_{1}^{\mathrm{w},<}$ already contain the most important features of the conductance. They approach each other and the classical limit with increasing tip strength, indicating that the effect of long trajectories is suppressed by strong tips.

The probabilities for reflected trajectories $P_{\mathrm{R}}^{\mathrm{b}}$ with an even number of bounces $b_{s}=\{0,2,4,6,8\}$ are presented in Fig. 5(b) [34]. The case of $b=0$ concerns the direct reflection from the tip without touching the cavity edge, as, e.g., the dashed red trajectory in Fig. 4(d). The weight of this class 


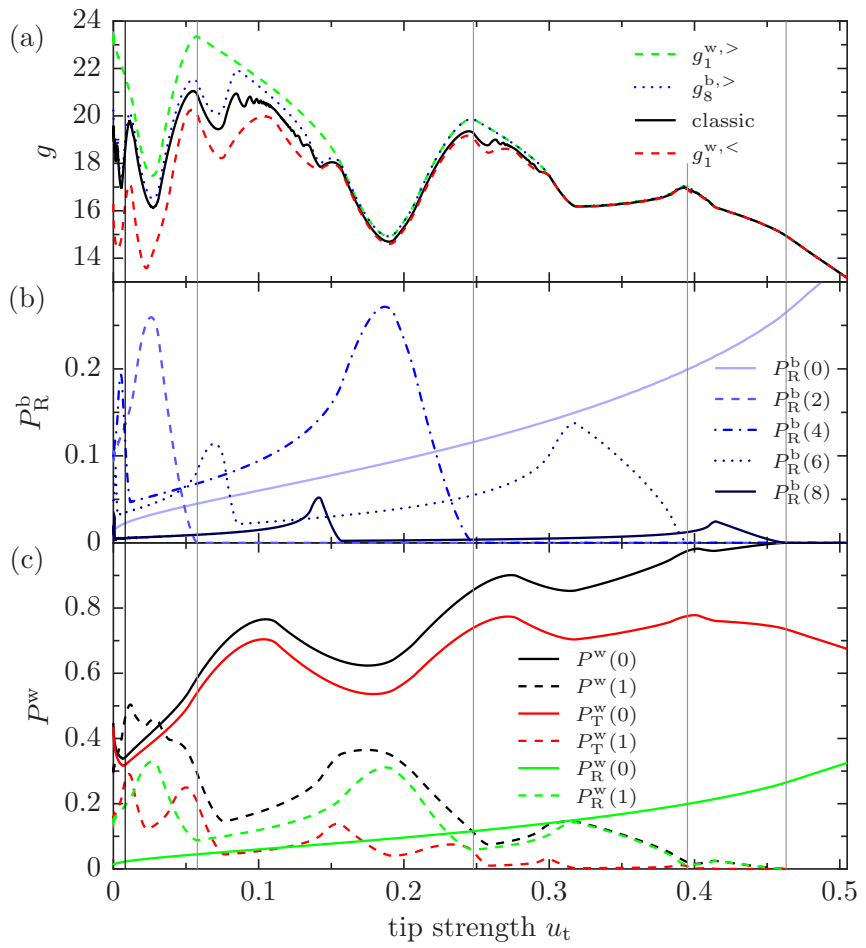

FIG. 5. Influence of tip strength on (a) the classical conductance and conductance bounds obtained with $g_{8}^{\mathrm{b},>}, g_{1}^{\mathrm{w},>}$, and $g_{1}^{\mathrm{w},<}$. (b) Probabilities $P_{\mathrm{R}}^{\mathrm{b}}$ of an electron to be reflected after $b_{s}=\{0,2,4,6,8\}$ bounces with the cavity wall. (c) Probabilities $P^{\mathrm{w}}$ of trajectories without (solid) and one winding (dashed) around the center of the cavity (black) and its reflected $P_{\mathrm{R}}^{\mathrm{w}}$ (green, light) and transmitted $P_{\mathrm{T}}^{\mathrm{w}}$ (red, medium) parts. The leftmost vertical line indicates the tip strength above which transmitted trajectories without bounces are impossible, and the following vertical lines indicate values above which reflected trajectories with two, four, six, and eight bounces are impossible.

of trajectories $P_{\mathrm{R}}^{\mathrm{b}}(0)$ grows with tip strength due to the increasing bending of the trajectories. For the same reason, the probability to be transmitted increases for trajectories that enter the cavity, hence reflected trajectories with a finite number of bounces are suppressed. The tip strengths where reflected trajectories with $b_{s}=\{2,4,6\}$ disappear (vertical lines in Fig. 5) are very close to the conductance maxima. We conclude that the disappearance of such a category of reflected trajectories is related to a maximum in the transmission. This is an important element of the mechanism underlying the observed large-scale conductance oscillations. Consistent with our previous findings, trajectories with $b_{s} \geqslant 8$ have little effect on the behavior of the conductance.

To illustrate the special role of the tip in selecting certain classes of transmitted or reflected trajectories, we discuss the example of trajectories with $b_{s}=2$ [dashed red curve in Fig. 4(b)]. For the reflected trajectories, $\Delta \varphi$ must satisfy $2 \pi-$ $2 \varphi_{\text {op }}<3 \Delta \varphi<2 \pi+2 \varphi_{\text {op }}$, where $\varphi_{\text {op }}=\arcsin \left(W / 2 R_{0}\right)$ is the opening angle of the contacts [see the inset of Fig. 2(b)]. With increasing tip strength, $\Delta \varphi$ decreases, and beyond a certain value the reflected trajectories with $b_{s}=2$ no longer exist. Concomitantly, the transmitted trajectories with $b_{s}=1$, where $\pi-2 \varphi_{\mathrm{op}}<2 \Delta \varphi<\pi+2 \varphi_{\mathrm{op}}$, gain importance. The blue solid line in Fig. 4(c) is an example of such a trajectory at $u_{\mathrm{t}}=0.06$. The structure of the current density for this regime of tip strength shown in Fig. 3 demonstrates that the quantum current flow is closely related to the shape of those transmitted trajectories. Reflection after a full winding is now only possible for trajectories with at least four bounces with the cavity wall [see the red dashed line in Fig. 4(c)]. This scenario is confirmed by the probabilities for zero and one windings $P^{\mathrm{w}}(0)$ and $P^{\mathrm{w}}(1)$ shown in Fig. 5(c). The number of transmitted trajectories with no winding around the center $P_{\mathrm{T}}^{\mathrm{w}}(0)$ is increasing, while the probability to find a reflected trajectory with one winding $P_{\mathrm{R}}^{\mathrm{w}}(1)$ assumes minima close to the vertical lines. Hence, the decrease of the weight of longer trajectories [a very long one is shown by the blue solid curve in Fig. 4(b)] with tip strength, and the alternation in the suppression of reflected and transmitted families, explains the oscillations of the conductance.

In general, the reduced $\Delta \varphi$ increases the probabilities to be either reflected without collision or to be transmitted after an increasing number of bounces but without a full winding around the cavity center. This is illustrated in Fig. 4(d) showing three trajectories at tip strength $u_{\mathrm{t}}=0.43$, where reflected trajectories with one winding and $b_{s}=8$ are still possible (see the dotted red line). Once the tip strength imposes $\Delta \varphi<2 \varphi_{\mathrm{op}}$, the bending of the trajectories becomes so strong that all trajectories are either immediately reflected, as shown by the dashed red curve in Fig. 4(d), or transmitted after $b_{s} \geqslant 3$ collisions, as shown by the blue solid curve in Fig. 4(d). Trajectories with a nonzero winding number become impossible, and $P^{\mathrm{w}}(0)=1$, such that there is no conductance maximum at the tip strength where reflection after eight bounces becomes impossible.

With a further increase of tip strength, the increase of direct reflection $P_{\mathrm{R}}^{\mathrm{w}}(0)$ continues, implying a decrease of the transmissions $P_{\mathrm{T}}^{\mathrm{w}}(0)$, and the conductance decreases monotonically with tip strength, reaching zero when the tip-induced depletion is so strong that electrons cannot enter the cavity anymore. Beyond the behavior accessible by our classical analysis, the quantum conductance in this regime exhibits quantized values that are due to the two parallel quantum wires that are formed between the cavity wall and the tip.

The initial decrease and the first minimum of the conductance at $u_{\mathrm{t}} \approx 0.01$ are due to the suppression of direct transmission without ever touching the cavity edge. Above the tip strength where direct trajectories cease to exist (leftmost vertical line in Fig. 5), the tip is no longer perturbative in the quantum-mechanical sense [35].

\section{SIMPLIFIED ANALYSIS OF THE CLASSICAL TRAJECTORIES}

The previous statistical analysis shows that a reduced subensemble of classical trajectories suffices to explain the dependence of the conductance on tip strength, and it points to the crucial importance of the parameter $\Delta \varphi$ that characterizes the angular distance between subsequent points where the trajectory hits the cavity boundary. In this section, we investigate the distribution of $\Delta \varphi$ at a given tip strength. An analytically tractable analysis based on the dominating trajectories provides the essence of the mechanism underlying 


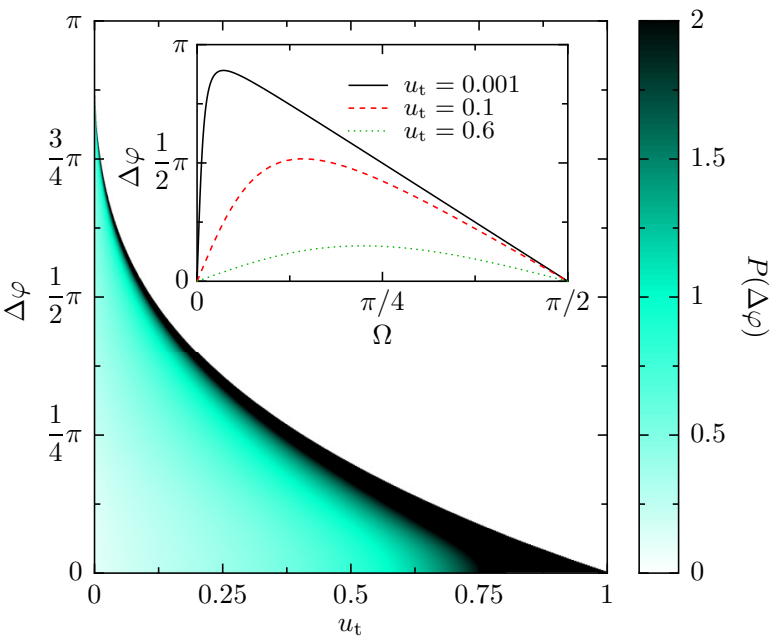

FIG. 6. Density plot of the probability distribution $P(\Delta \varphi)$ as a function of $u_{\mathrm{t}}$. Inset: dependence of $\Delta \varphi$ on the injection angle $\Omega$ for different values of $u_{\mathrm{t}}$.

the classical conductance oscillations, as well as the recipe to predict the main conductance maxima and minima. This is particularly useful since each of the previously analyzed subensembles of classical trajectories is actually infinite and mixes very different behaviors.

Figure 6 shows a density plot of the probability distribution of the rotation angle $P(\Delta \varphi)$ within our ensemble of trajectories for values of $u_{\mathrm{t}}$ between 0 and 1 . For a given $u_{\mathrm{t}}$, the angle $\Delta \varphi$ is solely a function of the angle $\Omega$ (due to symmetry, $\Delta \varphi$ does not depend on $\left.\varphi_{0}\right)$. The $\Omega$ dependence exhibits a maximum value $\Delta \varphi_{\mathrm{m}}$ (see Fig. 6, inset), which decreases with increasing tip strength. Figure 6 shows that the probability density is highly concentrated for values of $\Delta \varphi$ close to $\Delta \varphi_{\mathrm{m}}$, actually diverging when the maximum value of $\Delta \varphi_{\mathrm{m}}$ is approached from below. This behavior is due to the long tails of the tip potential (2) that lead to a smooth dependence of $\Delta \varphi$ on the injection angle $\Omega$ defined with respect to the cavity diameter [see Fig. 4(a)]. Trajectories injected with $\Omega=0$ are reflected in a head-on collision with the tip and have $\Delta \varphi=0$. Then, $\Delta \varphi$ reaches its maximum value $\Delta \varphi_{\mathrm{m}}$ at an intermediate value of $\Omega$, and it decreases upon a further increase of $\Omega$ back to 0 when $\Omega=\pi / 2$. Examples of this behavior are shown in the inset of Fig. 6. The flat maximum occurring in the dependence of $\Delta \varphi$ on $\Omega$ leads to the divergence of $P(\Delta \varphi)$ at the maximum value $\Delta \varphi_{\mathrm{m}}$.

The resulting dominance of trajectories with $\Delta \varphi$ close to the maximum value $\Delta \varphi_{\mathrm{m}}$ becomes more pronounced with increasing values of $u_{\mathrm{t}}$ (and decreasing $\Delta \varphi_{\mathrm{m}}$ ), and motivates the characterization of the ensemble of classical trajectories at a given tip strength by the dominant angular distance between bounces $\Delta \varphi_{\mathrm{m}}$. We restrict the analysis in the sequel to only those trajectories having $\Delta \varphi=\Delta \varphi_{\mathrm{m}}$.

In the regime, where the tip is so strong that $\Delta \varphi_{\mathrm{m}}<2 \varphi_{\mathrm{op}}$, all trajectories are reflected immediately without ever hitting the cavity wall, with the exception of those that start at a position that is separated by less than $\Delta \varphi_{\mathrm{m}}$ from the edge of the opening $\varphi_{\mathrm{op}}$. The latter trajectories are transmitted to the other lead after a number of bounces, but without winding around the center. Their proportion is given by $\Delta \varphi_{\mathrm{m}} / 2 \varphi_{\mathrm{op}}$ such

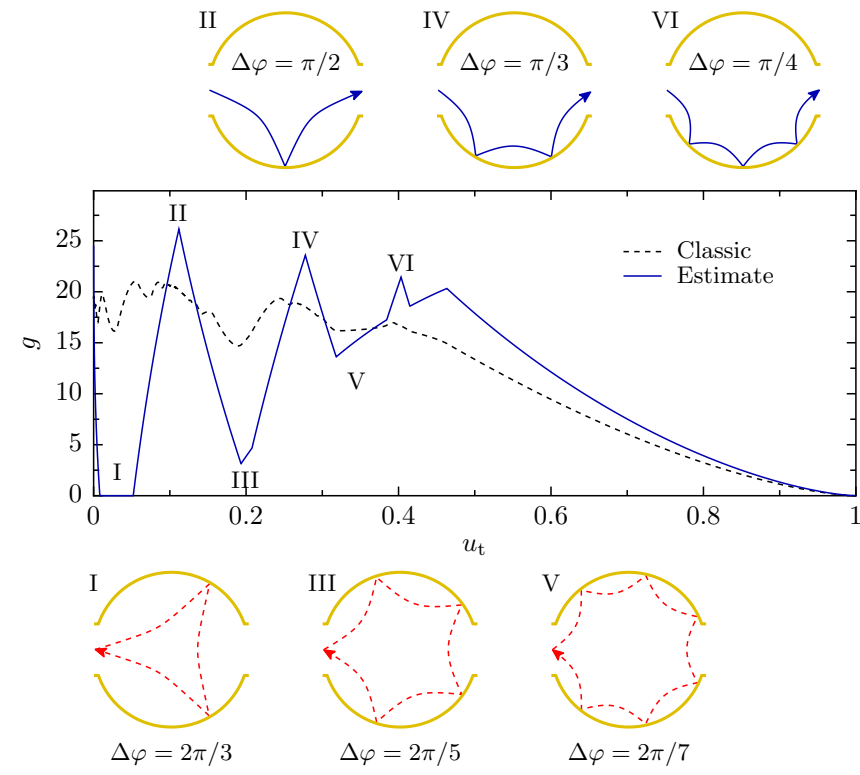

FIG. 7. Conductance estimates (11) and (12) based on the shortest trajectories with maximum $\Delta \varphi$ (blue solid line). The classical conductance (black dashed line) is shown for comparison. Sketches of the dominant classes of short transmitted and reflected trajectories in the regions of conductance maxima and minima are shown above and below the plot, respectively.

that the classical conductance is estimated as

$$
g_{\text {class }} \approx \frac{m v_{0} W}{\hbar \pi} \frac{\Delta \varphi_{\mathrm{m}}}{2 \varphi_{\mathrm{op}}} .
$$

The decrease with $u_{\mathrm{t}}$ is a consequence of the increasing number of directly reflected trajectories when $\Delta \varphi_{\mathrm{m}}$ is suppressed by an increasing tip strength.

For weaker tip strength, when $\Delta \varphi_{\mathrm{m}}>2 \varphi_{\mathrm{op}}$, the distance between bounces is larger than the openings of the cavity, and a direct reflection is impossible. Due to the relatively large openings of the cavity, longer trajectories are of minor importance, especially for large $u_{\mathrm{t}}$. For simplicity, we neglect them and concentrate on trajectories with no winding and transmitted after $b$ bounces. For such trajectories, the total rotation angle $(b+1) \Delta \varphi$ lies in the interval $\left[\pi-2 \varphi_{\mathrm{op}}, \pi+2 \varphi_{\mathrm{op}}\right]$. The evaluation of the share of initial positions in the left contact that lead to such a trajectory yields the estimate

$$
g^{\mathrm{b}}(b) \approx \frac{m v_{0} W}{\hbar \pi}\left(1-\frac{\left|\pi-(b+1) \Delta \varphi_{\mathrm{m}}\right|}{2 \varphi_{\mathrm{op}}}\right)
$$

for their contribution to the conductance. Thus, maxima of the total conductance, obtained by summing over $b$, can be expected when $\Delta \varphi_{\mathrm{m}}=\pi /(b+1)$. They are of triangular shape with a width $4 \varphi_{\mathrm{op}} /(b+1)$.

For the simplified model with the tip potential (2), the above criterion for transmission maxima translates into conductance peaks at $u_{\mathrm{t}} \approx\{0,0.111,0.278,0.403\}$ (blue solid curve in Fig. 7). Close to these values, maxima arise in the probability of having a transmitted trajectory with zero winding [red solid line in Fig. 5(c)]. The estimates of the conductance (11) 
and (12) that are based on the shortest transmitted trajectories are represented by the blue solid line in Fig. 7 that can be compared to the classical conductance (black dashed line). The classes of short transmitted trajectories that dominate in the vicinity of the conductance peaks are sketched above the plot. The qualitative agreement of the peak structure confirms the dominance of those short trajectories. However, at small tip strength $u_{\mathrm{t}}$ the quantitative comparison becomes rather poor, pointing to the increased importance of longer trajectories and a broader distribution of the values of $\Delta \varphi$.

The geometric argument can be extended to longer trajectories. The class of trajectories that are reflected after one winding around the cavity lead to a minimum in conductance at the values $\Delta \varphi_{\mathrm{m}}=2 \pi /(b+1)$ with the width $4 \varphi_{\mathrm{op}} /(b+1)$ for even $b$. The corresponding conductance minima are expected at $u_{\mathrm{t}} \approx\{0.031,0.199,0.345,0.425\}$. These values are close to the minima in the classical conductance (Fig. 7), as well as to the maxima in the probability of having reflected trajectories with one winding [green dashed line in Fig. 5(c)]. Also, the probabilities $P_{\mathrm{R}}^{\mathrm{b}}(b)$ of being reflected after $b$ bounces in Fig. 5(b) assume maxima close to the corresponding values of $u_{\mathrm{t}}$. The dominant classes of short reflected trajectories close to the conductance minima are sketched below the graph in Fig. 7.

The long-range tip potential is a crucial ingredient for the mechanism presented above since it leads to the dominance of trajectories at $\Delta \varphi_{\mathrm{m}}$. In contrast, when the tip is modeled by a hard wall disk, the dependence of $\Delta \varphi$ on the injection angle has a cusp at the maximum. The resulting probability density does not exhibit a significant preferential value, the behavior of very different values of $\Delta \varphi$ is mixed, and the conductance oscillations are smeared, consistent with our numerical observations. The role of the steepness of the cavity potential is very different as it does not affect significantly the specular reflection at the cavity edges. Thus, assuming hard wall boundaries for the cavity is a very good approximation, at least within the present study.

\section{OFF-CENTER TIP}

The experimental SGM scans of Figs. 1(b) and 1(c) show that the nonmonotonic behavior of the conductance as a function of tip strength extends to a well-defined region around the center of the cavity. It is important to understand the robustness of this surprising effect, since moving the tip off-center has the important consequence of breaking the integrability of the underlying classical dynamics.

In Fig. 8 we present cuts of the experimental data of Fig. 1(b) corresponding to the tip voltage $V_{\text {tip }}=-3 \mathrm{~V}$ for the two axis directions $x$ and $y$, together with the numerical simulation within the realistic model. The agreement between the two sets of data is quite good. The deviations obtained for tips approaching the border of the cavity are probably due to our approximation of superposing the tip-induced potential and the confinement potential. The small-scale structure in the numerical traces is less pronounced in the experiment, where inelastic processes suppress the contribution of long trajectories.

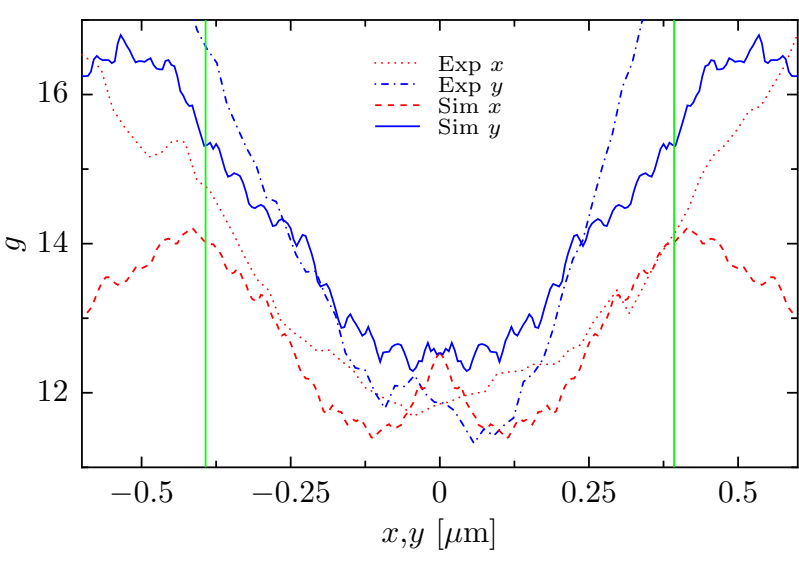

FIG. 8. Conductance cuts as a function of tip position in $x$ (red) and $y$ (blue) directions away from the cavity center. Solid and dashed lines represent the realistic numerical simulation at $u_{\mathrm{t}}=0.0315$ and $T=300 \mathrm{mK}$, while dash-dotted and dotted lines represent the experimental conductance at $V_{\text {tip }}=-3 \mathrm{~V}$. The green vertical lines represent the rough estimate $y / R_{0} \approx \pi / 12$ for the size of the central spot in Fig. 1(b) (see the text).

It is important to remark that the numerical calculations reproduce the width of the conductance dip observed in the cavity center and even the asymmetry of the central spot in Fig. 1(b), indicating a faster recovery of high conductance values when moving away from the center in the $y$ direction as compared with the $x$ direction. As in the previously studied case of a centered tip, the physical understanding of the spatial extension of the nonmonotonic dependence of conductance on tip voltage requires the study of the simplified model and a semiclassical analysis. The numerics in the simplified model (not shown) is consistent with the experimental results and the spatial anisotropy. Within the simplified analysis in terms of classical trajectories, we can make a rough estimate of the size of the central spot in Fig. 1(b).

The minimum conductance observed at $V_{\text {tip }}=-3 \mathrm{~V}$ is due to the dominance of reflected "triangular" trajectories with $b=2$ bounces and $w=1$ winding (see Fig. 5) that are most prominent when $\Delta \varphi_{\mathrm{m}} \approx 2 \pi / 3$. The subsequent conductance maximum corresponds to the dominance of trajectories with $b=1$ and $w=0$. Those trajectories correspond to $\Delta \varphi_{\mathrm{m}} \approx$ $\pi / 2$ that are transmitted after a single bounce at the cavity edge, and that can pass above or below the tip. We assume that those short trajectories, although deformed, still determine the behavior when the tip is slightly off-centered. For a displacement in the $y$ direction, the angular distance of the bounces seen from the tip position is modified to $\Delta \varphi \approx \pi / 2 \pm$ $y / R_{0}$. Assuming that the conductance oscillations are washed out when the resulting difference of $2 y / R_{0}$ in $\Delta \varphi$ between trajectories above and below the tip reaches the difference $2 \pi / 3-\pi / 2$ corresponding to the conductance maximum and minimum, we get the estimate $y / R_{0} \approx \pi / 12$ for the distance $y$ between the tip and the cavity center in the transverse direction where the classical oscillations are expected to be smeared. This universal value, independent of the size of the cavity openings, is indicated by the green vertical lines in Fig. 8. It is consistent with the experimental findings [the size of the 
central region of suppressed conductance in Fig. 1(b)] and also with our numerical calculations.

\section{CONCLUSIONS}

We presented SGM experiments on a circular cavity in which an intriguing nonmonotonic dependence of the conductance on the tip voltage is observed when the tip is placed near the center of the cavity. Our theoretical analysis explains the unexpected behavior and traces it back to classical electron dynamics in the device.

The numerical simulation of quantum transport through the experimental setup with a long-range tip potential in the center at the temperature of the experiment yielded large conductance oscillations as a function of tip strength that are in quantitative agreement with the experimental findings [see Fig. 2(a)]. At low temperature, additional ballistic conductance fluctuations appear in the numerically calculated conductance.

The quest for a physical understanding of the surprising nonmonotonic behavior found in the experiments and in the realistic quantum simulations led us to proceed in three steps. First, we simplified the model in order to have, at the same time, the quantum simulations exhibiting the observed behavior, and an analytically tractable classical dynamics. Second, we performed a statistical analysis of short classical trajectories that linked the conductance oscillation with the switches between groups of dominant trajectories. Third, we developed a simplified analysis that identified the specific features of the trajectories that result in the experimentally observed phenomenon.

While the quantum conductance fluctuations are suppressed at the temperature of the experiment, the classical conductance is not significantly affected by temperature. The large-scale conductance oscillations obtained in the classical limit reproduce the behavior of the quantum conductance at the experimental temperature, except for conductance steps that arise in the regime of very strong tips. We therefore conclude that the oscillations of the conductance as a function of tip strength and the resulting nonmonotonic behavior of the conductance when the tip is in the center of the cavity are of classical origin.

The statistical analysis of the ensemble of trajectories, possible in the simplified model system, shows that relatively short trajectories with few bounces with the cavity wall or few windings around the cavity center allow us to understand the conductance oscillations. The evolution of the contributing trajectories with tip strength provides the mechanism for the observed conductance oscillations.

Having observed that trajectories with a particular angular distance between bounces at the cavity wall dominate in the case of strong tip potentials, we showed that the restriction to this class of dominating trajectories leads to a basic understanding of the main conductance maxima. We found that the long-range character of the tip potential is a crucial ingredient for the classical mechanism. When the tip is modeled as a hard disk of increasing size, the experimentally observed nonmonotonic behavior of the conductance cannot be reproduced.

The classical analysis presented in this work is able to account for the conductance oscillations as a function of the tip strength for the case of centered tips, but also for the experimentally observed decay of these oscillations as the tip moves away from the cavity center.

It is remarkable that a simple modeling based on relatively short classical trajectories, completely ignoring disorder and electron-electron interaction, was capable of rendering the explanation of the measured conductance through a cavity within an SGM setup. Even if the modeling of ballistic transport is usually done for quantum billiards, the role of the smoothness of the electrostatic confinement potential has been discussed in the literature [36,37]. In our work, we have shown that the electrostatic confinement defining the structure seems to be sufficiently sharp so as not to give rise to important departures from the hard-wall case. However, the smooth character of the electrostatic potential created by the tip imprints a crucial signature for the electric transport with an SGM setup.

The role of short trajectories in ballistic transport has been pointed out in the context of various experimental setups [17]. In particular, it has been shown [38] that for classically chaotic cavities, the deterministic sector of the phase space corresponding to short trajectories may give rise to conductance oscillations that are more pronounced than the conductance fluctuations stemming from the stochastic sector. The relative weight of the deterministic and stochastic sectors of phase space can be changed by varying the openings of the cavity, or, within a fixed geometry, by the application of a magnetic field (as in experiments measuring shot noise in chaotic cavities [39]).

While the signature of classical trajectories in transport through circular billiards has been identified $[17,19,40]$ by the Fourier transform of the energy and magnetic-field-dependent conductance, our experimental and theoretical results provide direct evidence of the almost exclusive role of a small class of trajectories. The correlation of magnetoresistance maxima with specific electron trajectories has been observed in the case of triangular [41] and circular [42] cavities under a perpendicular magnetic field. The commensurability conditions between the cyclotron radius and the linear dimensions of the cavities result in magnetoconductance oscillations similar to the conductance oscillations that we study in this work. Our case presents the particularity that the curvature of the classical trajectories depends on the impinging angle, resulting in the domination of a given rotation angle between successive bounces with the walls of the cavity, leading to very large conductance oscillations.

Experiments carried out in small cavities (diameter 1.0 $1.5 \mu \mathrm{m})$ do not show significant traces of the classical oscillations as compared to those of the large cavity of Fig. 1. The small cavities do not have perfect circular symmetry, which might explain the different behavior. Moreover, numerical simulations indicate a stronger difference between the Lorentzian and the simplified tip potential for the case of the smaller cavities, and they point to a modification of the mechanisms in the case in which the width of the Lorentzian tip potential is not much smaller than the cavity.

In the small cavities, it is possible to achieve the regime in which the size of the tip-induced disk becomes comparable with the size of the cavity [27,43]. SGM measurements in this regime show fringes that extend to the center of the 
cavity and a conductance suppression with increasing negative tip voltage exhibiting steps. Such behavior is consistent with the conductance quantization at large tip strength that can be observed in the result for the quantum conductance presented in Fig. 2.

\section{ACKNOWLEDGMENTS}

Financial support from the French National Research Agency ANR (Projects ANR-11-LABX-0058_NIE and ANR14-CE36-0070-01) and from the Swiss National Science Foundation SNSF is gratefully acknowledged.
[1] M. A. Topinka, B. J. LeRoy, S. E. J. Shaw, E. J. Heller, R. M. Westervelt, K. D. Maranowski, and A. C. Gossard, Science 289, 2323 (2000).

[2] M. A. Topinka, B. J. LeRoy, R. M. Westervelt, S. E. J. Shaw, R. Fleischmann, E. J. Heller, K. D. Maranowski, and A. C. Gossard, Nature (London) 410, 183 (2001).

[3] B. J. LeRoy, A. C. Bleszynski, K. E. Aidala, R. M. Westervelt, A. Kalben, E. J. Heller, S. E. J. Shaw, K. D. Maranowski, and A. C. Gossard, Phys. Rev. Lett. 94, 126801 (2005).

[4] E. J. Heller, K. E. Aidala, B. J. LeRoy, A. C. Bleszynsky, A. Kalben, R. M. Westervelt, K. D. Maranowski, and A. C. Gossard, Nano Lett. 5, 1285 (2005).

[5] M. P. Jura, M. A. Topinka, M. Grobis, L. N. Pfeiffer, K. W. West, and D. Goldhaber-Gordon, Phys. Rev. B 80, 041303 (2009).

[6] M. P. Jura, M. Grobis, M. A. Topinka, L. N. Pfeiffer, K. W. West, and D. Goldhaber-Gordon, Phys. Rev. B 82, 155328 (2010).

[7] S. Schnez, J. Güttinger, C. Stampfer, K. Ensslin, and T. Ihn, New J. Phys. 13, 053013 (2011).

[8] H. Sellier, B. Hackens, M. G. Pala, F. Martins, S. Baltazar, X. Wallart, L. Desplanque, V. Bayot, and S. Huant, Semicond. Sci. Technol. 26, 064008 (2011).

[9] F. Martins, B. Hackens, M. G. Pala, T. Ouisse, H. Sellier, X. Wallart, S. Bollaert, A. Cappy, J. Chevrier, V. Bayot, and S. Huant, Phys. Rev. Lett. 99, 136807 (2007).

[10] R. A. Jalabert, W. Szewc, S. Tomsovic, and D. Weinmann, Phys. Rev. Lett. 105, 166802 (2010).

[11] C. Gorini, R. A. Jalabert, W. Szewc, S. Tomsovic, and D. Weinmann, Phys. Rev. B 88, 035406 (2013).

[12] R. Steinacher, A. A. Kozikov, C. Rössler, C. Reichl, W. Wegscheider, K. Ensslin, and T. Ihn, Phys. Rev. B 93, 085303 (2016).

[13] A. A. Kozikov, D. Weinmann, C. Rössler, T. Ihn, K. Ensslin, C. Reichl, and W. Wegscheider, Phys. Rev. B (to be published).

[14] A. A. Kozikov, D. Weinmann, C. Rössler, T. Ihn, K. Ensslin, C. Reichl, and W. Wegscheider, New J. Phys. 15, 083005 (2013).

[15] M. G. Pala, S. Baltazar, P. Liu, H. Sellier, B. Hackens, F. Martins, V. Bayot, X. Wallart, L. Desplanque, and S. Huant, Phys. Rev. Lett. 108, 076802 (2012).

[16] A. A. Sousa, A. Chaves, G. A. Farias, and F. M. Peeters, Phys. Rev. B 88, 245417 (2013).

[17] R. A. Jalabert, Scholarpedia 11, 30946 (2016).

[18] C. M. Marcus, A. J. Rimberg, R. M. Westervelt, P. F. Hopkins, and A. C. Gossard, Phys. Rev. Lett. 69, 506 (1992).

[19] M. J. Berry, J. A. Katine, R. M. Westervelt, and A. C. Gossard, Phys. Rev. B 50, 17721 (1994).

[20] M. Persson, J. Pettersson, B. von Sydow, P. E. Lindelof, A. Kristensen, and K. F. Berggren, Phys. Rev. B 52, 8921 (1995).

[21] A. M. Chang, H. U. Baranger, L. N. Pfeiffer, and K. W. West, Phys. Rev. Lett. 73, 2111 (1994).

[22] Y. Lee, G. Faini, and D. Mailly, Phys. Rev. B 56, 9805 (1997).
[23] A similar nonintuitive behavior has been found when blocking one of the paths of a multiply connected geometry, and it has been interpreted as an electronic analog of the Braess paradox $[15,16]$. Such a quantum phenomenon has a very different origin from the classical mechanism underlying the conductance oscillations that we observe.

[24] S. E. J. Shaw, Ph.D. thesis, Harvard University, 2002.

[25] Such a choice affects the value of the dimensionless strength parameter $u_{\mathrm{t}}$, but it does not imply that the tip potential $U_{\mathrm{S}}(\vec{r})$ depends on the Fermi energy or the size of the cavity.

[26] C. W. Groth, M. Wimmer, A. R. Akhmerov, and X. Waintal, New J. Phys. 16, 063065 (2014).

[27] R. Steinacher, A. A. Kozikov, C. Rössler, C. Reichl, W. Wegscheider, T. Ihn, and K. Ensslin, New J. Phys. 17, 043043 (2015).

[28] A harmonic tip potential fails to produce large-scale oscillations with a sufficient amplitude, and assuming a Coulomb-like tip potential $U_{\mathrm{t}}(\vec{r}) \propto u_{\mathrm{t}} /\left(\vec{r}-\vec{r}_{T}\right)$ leads to qualitatively different oscillations.

[29] R. Blümel and U. Smilansky, Phys. Rev. Lett. 60, 477 (1988).

[30] R. A. Jalabert, H. U. Baranger, and A. D. Stone, Phys. Rev. Lett. 65, 2442 (1990).

[31] H. U. Baranger, D. P. DiVincenzo, R. A. Jalabert, and A. D. Stone, Phys. Rev. B 44, 10637 (1991).

[32] Spatial symmetries induce action degeneracies among the trajectories contributing to (4), which result in quantum corrections to the classical transmission probability [17]. For chaotic geometries, these corrections are typically of the order $2 \times e^{2} / h$ [44]. In particular, the reflection symmetry with respect to the $x$ axis does not alter the prescription (4) [31]. The case of integrable geometries is more complicated due to the quasidegeneracy of actions within families of trajectories. In our problem, we do not expect the corresponding corrections to be relevant since long trajectories are of minor importance. Moreover, changing tip strength does not affect the symmetries. For the large openings of the cavity under study, the diffractive corrections to the semiclassical approach [45] are irrelevant.

[33] Choosing the starting points on the circle of radius $R_{0}$ leads to a small deviation with respect to the prescription of Eq. (4). In addition, the tip potential affects the initial velocities [31]. Though it is in principle possible to take these two corrections into account, we have checked that for not too strong tip strength, their effect on the conductance and the statistics of the trajectories is very small and can be neglected in the regime in which we are interested.

[34] The symmetry of the system, with equal width openings exactly opposite to each other, makes reflection after an odd number of bounces impossible, such that $P_{\mathrm{R}}^{\mathrm{b}}(b)$ vanishes for all odd $b$, independent of the tip strength. 
[35] First-order perturbation theory of SGM [10,11] can only predict the initial slope in the curve of the conductance versus tip strength.

[36] T. Ouchterlony, I. V. Zozoulenko, C.-K. Wang, K.-F. Berggren, C. Gould, and A. S. Sachrajda, Eur. Phys. J. B 10, 361 (1999).

[37] C. A. Marlow, R. P. Taylor, T. P. Martin, B. C. Scannell, H. Linke, M. S. Fairbanks, G. D. R. Hall, I. Shorubalko, L. Samuelson, T. M. Fromhold, C. V. Brown, B. Hackens, S. Faniel, C. Gustin, V. Bayot, X. Wallart, S. Bollaert, and A. Cappy, Phys. Rev. B 73, 195318 (2006).

[38] P. Jacquod and E. V. Sukhorukov, Phys. Rev. Lett. 92, 116801 (2004).

[39] S. Oberholzer, E. V. Sukhorukov, and C. Schönenberger, Nature (London) 415, 765 (2002).
[40] H. Ishio and J. Burgdörfer, Phys. Rev. B 51, 2013 (1995).

[41] H. Linke, L. Christensson, P. Omling, and P. E. Lindelof, Phys. Rev. B 56, 1440 (1997).

[42] B. Hackens, F. Delfosse, S. Faniel, C. Gustin, H. Boutry, X. Wallart, S. Bollaert, A. Cappy, and V. Bayot, Phys. Rev. B 66, 241305 (2002).

[43] A. A. Kozikov, R. Steinacher, C. Rössler, T. Ihn, K. Ensslin, C. Reichl, and W. Wegscheider, New J. Phys. 16, 053031 (2014).

[44] R. S. Whitney, H. Schomerus, and M. Kopp, Phys. Rev. E 80, 056209 (2009).

[45] I. Březinová, L. Wirtz, S. Rotter, C. Stampfer, and J. Burgdörfer, Phys. Rev. B 81, 125308 (2010). 